\title{
Estimating the Potential Impact of CYP2C19 and CYP2D6 Genetic Testing on Protocol-Based Care for Depression in Canada and the United States
}

\author{
Mikayla Fan ${ }^{a}$ Chad A. Bousman ${ }^{\text {b-d }}$ \\ ${ }^{a}$ Cumming School of Medicine, University of Calgary, Calgary, AB, Canada; bepartments of Medical Genetics, \\ Psychiatry, Physiology \& Pharmacology, University of Calgary, Calgary, AB, Canada; ' Alberta Children's Hospital \\ Research Institute, Calgary, AB, Canada; ${ }^{d}$ Hotchkiss Brain Institute, Cumming School of Medicine, University of \\ Calgary, Calgary, $A B$, Canada
}

\section{Keywords}

Pharmacogenetics - STAR*D - Major depressive disorder ·

Antidepressants

\begin{abstract}
The Sequenced Treatment Alternatives to Relieve Depression (STAR*D) algorithm is the most recognized protocolbased care approach for moderate to severe depression. However, its implementation results in one-third of individuals receiving modest to no symptom remission. One possible explanation is the inter-individual differences in antidepressant metabolism due to CYP2C19 and CYP2D6 genetic variation. Here, we aimed to determine the potential benefit of pairing CYP2C19 and CYP2D6 testing with the five-step STAR*D algorithm. To estimate the proportion of individuals that could benefit from CYP2C19 and CYP2D6 testing, we simulated the STAR*D algorithm using ethnicity-specific phenotype (e.g., metabolizer status) frequencies published by the Clinical Pharmacogenetics Implementation Consortium and census data from the Canada and the US. We found that up to one-third of the US and Canadian populations being treated for depression could benefit from the addition of CYP2C19 and CYP2D6 genetic testing. The potential benefit
\end{abstract}

๑) 2019 S. Karger AG, Basel varied for each step of the algorithm and for each province, territory, and state. CYP2C19 genotyping had the greatest potential impact within the first two steps of the algorithm, while CYP2D6 genotyping had the most notable impact in Steps 3, 4, and 5. Our findings suggest the implementation of CYP2C19 and CYP2D6 genetic testing alongside the $S T A R * D$ treatment algorithm may improve depression treatment outcomes in Canada and the US.

(c) 2019 S. Karger AG, Basel

\section{Introduction}

Numerous treatment algorithms have been developed to assist clinicians in providing protocol-based care for moderate to severe depression. The Sequenced Treatment Alternatives to Relieve Depression (STAR*D) algorithm is the most well-known protocol-based care approach, consisting of five sequential steps of treatment options [1]. All individuals begin at Step 1 with citalopram. If adequate response is not achieved, individuals are encouraged to proceed to Step 2, where citalopram therapy is augmented (bupropion, buspirone, or cognitive therapy) or switched to another antidepressant (bu- 
propion-SR, sertraline, or venlafaxine). Likewise, in Steps $3-5$, those with inadequate response have their therapy switched (nortriptyline, mirtazapine, bupropion, venlafaxine, tranylcypromine, or venlafaxine+mirtazapine) or augmented (lithium or T3) until remission is achieved or they exit from the algorithm (e.g., loss-to-follow-up). Randomized clinical trial findings have showed implementation of the STAR*D algorithm results in about twothirds of individuals with depression achieving symptom remission, while the remaining one-third received modest to no symptom relief [2]. However, it remains unclear why the algorithm fails to get more individuals well.

One explanation for the algorithms less than optimal performance is the presence of inter-individual variation in genes involved in antidepressant metabolism. In fact, a previous genetic analysis of the STAR* $\mathrm{D}$ trial data suggested citalopram (Step 1) tolerance and response was associated with genetic variation in CYP2C19 but not $C Y$ $P 2 D 6$ [3], two cytochrome P450 enzymes involved in the phase 1 metabolism of most antidepressants. However, the potential effect of CYP2C19 and CYP2D6 genetic variation on tolerance and response in subsequent steps of the STAR*D algorithm has not been evaluated. This is particularly important given that at each of the five steps of the STAR*D algorithm, there are medications (i.e., citalopram, sertraline, venlafaxine, nortriptyline) with associated CYP2C19- or CYP2D6-based prescribing guidelines [4-6]. Unfortunately, the STAR*D algorithm does not currently account for these guidelines or consider inter-individual variations in antidepressant metabolism.

To estimate the proportion of individuals that could potentially benefit from the inclusion of CYP2C19- and CYP2D6-based prescribing guidelines in the STAR*D algorithm, we undertook a population-based pharmacogenetic study using available data from Canadian provincial/territories and US states. We hypothesized that a considerable proportion of individuals could benefit from the inclusion of CYP2C19 and CYP2D6 genetic information in the STAR*D algorithm, and that the magnitude of this benefit would vary by province, territory, and state.

\section{Materials and Methods}

\section{Data Sources}

The most current population estimates by age and ethnicity for each Canadian province/territory and US state were obtained from the Statistics Canada website [7] and the US Census Bureau website [8], respectively. Frequencies of CYP2C19 and CYP2D6 genotype-predicted metabolizer phenotypes (i.e., poor, intermediate, normal, rapid/ultrarapid) by ethnicity were obtained from phenotype frequency tables available from the Clinical Pharmacogenet- ics Implementation Consortium (CPIC) [9]. CPIC determines $\mathrm{CY}$ P2D6 and CYP2C19 metabolizer phenotype based on the combination of star $(*)$ alleles present. Star alleles can represent a single variant or multiple variants (haplotype), each assigned a function (no, decreased, normal, increased, uncertain, unknown). For example, an individual with a CYP2C19 genotype of $* 1 /{ }^{*} 17$ would be defined as a rapid metabolizer because they carry one normal function allele $\left({ }^{*} 1\right)$ and one increased function allele $\left({ }^{*} 17\right)$. Whereas, an individual with a*2/*2 CYP2C19 genotype would be considered a poor metabolizer, given the presence of two no function alleles. Detailed explanations of star allele functions and their translation to metabolizer phenotypes can be found at the Pharmacogene Variation Consortium [10] (www.pharmvar.org) and CPIC [11] (cpicpgx.org) websites, respectively.

\section{Contraindicated Phenotype Estimation}

Prior to estimating the frequencies of CYP2C19 and CYP2D6 contraindicated phenotypes, each medication from the STAR*D algorithm was cross-referenced with guidelines developed by CPIC and the Dutch Pharmacogenetics Working Group (DPWG). At the time of our analysis, the only STAR*D medications with CYP2C19- and CYP2D6-based prescribing guidelines were citalopram, sertraline, venlafaxine, and nortriptyline $[4,5]$. Based on these guidelines, contraindicated phenotypes for citalopram and sertraline included CYP2C19 poor, rapid , and ultrarapid metabolizers, whereas for venlafaxine and nortriptyline contraindicated phenotypes included CYP2D6 poor, intermediate, and ultrarapid metabolizers. Next, each of the provincial/territory/state population datasets were restricted to individuals aged 18 years or older to reflect the population for which the STAR*D algorithm was designed. Then, the absolute number of individuals ( $\geq 18$ years old) in each US state that identified as Caucasian/European, African/ African-American, Asian, American (e.g., North American Indigenous origins, Indigenous from Central/South America), and Oceanian was extracted. Likewise, the absolute number of individuals in each Canadian province/territory that identified as Caucasian, American, African, Middle Eastern, South/Central Asian, East Asian, and Oceanian were extracted. These absolute numbers were then multiplied by the frequency of CYP2C19 or CYP2D6 contraindicated phenotypes for each ethnic group and then summed across ethnic groups to arrive at an estimated number of individuals with a contraindicated phenotype in each province/territory/ state for each of the four medications with CYP2C19- or CYP2D6based prescribing guidelines.

\section{Impact Estimation}

To estimate the potential impact of pairing CYP2C19 and $C Y$ $P 2 D 6$ genetic testing with the STAR*D algorithm, we simulated each step of the algorithm in each province/territory/state. These simulations began by multiplying each province/territory/state's total population by an estimated antidepressant use rate. Antidepressant prevalence rates were based on the most current national data available for each country. For Canadian provinces/territories a $9.1 \%$ antidepressant prevalence rate was applied [12], while for each US state a prevalence rate of $12.7 \%$ was used [13]. The estimated population using antidepressants was then entered into the STAR*D algorithm and the proportion of these individuals that would receive a treatment contraindicated for their CYP2C19 and/ or CYP2D6 phenotype was estimated at each step. Because Step 2 was the only step that included medications linked to CYP2C19
Fan/Bousman 


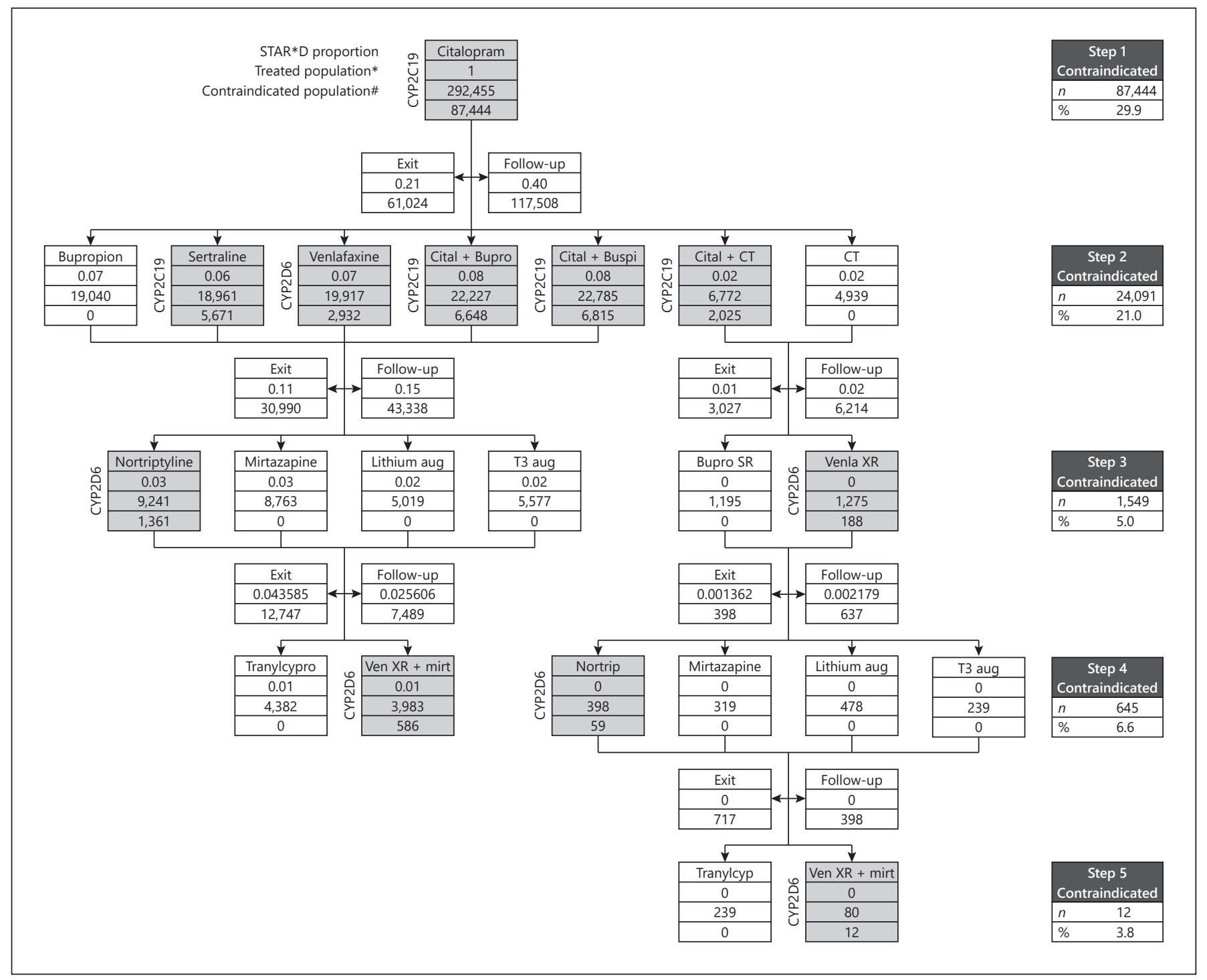

Fig. 1. Example of the simulation framework and parameters used to estimate the number and percentage of individuals that could benefit from CYP2C19 or CYP2D6 genetic testing at each step of the $\mathrm{STAR}^{*} \mathrm{D}$ algorithm within each province/territory/state. Grey boxes represent treatment options with a CYP2C19- or CYP2D6based prescribing guideline. ${ }^{*}$ The treated population was deter-

and CYP2D6 prescribing guidelines, we also estimated separately the proportion of those entering Step 2 that would benefit from CYP2C19 and CYP2D6 genotyping. Remission and exit rates at each step were based on the rates observed in the STAR*D trial [1]. Figure 1 provides an example of the simulation framework and parameters used to estimate the number and percentage of individuals that could benefit from CYP2C19 or CYP2D6 genetic testing at each step of the STAR*D algorithm within each province/ territory/state.

Impact of Pharmacogenetic Testing on Protocol-Based Care mined by multiplying the full adult population by the antidepressant prevalence rate $(0.091$ for Canadian provinces/territories; 0.127 for US states). " Contraindicated population is the estimated number of individuals that could benefit from $C Y P 2 C 19$ or $C Y$ $P 2 D 6$ genetic testing.

\section{Results}

The estimated proportion of individuals by ethnicity with a metabolizer phenotype contraindicated for the treatment received at each step of the STAR*D algorithm are presented in Table 1. Regardless of ethnicity, we found that the proportion of individuals that could benefit from $C Y$ P2C19 or CYP2D6 genotyping was highest at Steps 1 and 2 of the STAR*D algorithm but then considerably decreased 
Table 1. Estimated percentage of individuals with a CYP2C19 and/ or CYP2D6 phenotype contraindicated for the treatment received at each step of the STAR*D algorithm by ethnicity

\begin{tabular}{lccccc}
\hline Ethnicity & Step 1 & Step 2 & Step 3 & Step 4 & Step 5 \\
\hline Caucasian & 34.0 & 23.8 & 5.5 & 7.2 & 4.1 \\
American & 26.6 & 18.7 & 4.4 & 5.8 & 3.3 \\
African & 20.7 & 16.1 & 6.4 & 8.5 & 4.8 \\
African-American & 31.7 & 23.0 & 6.6 & 8.8 & 4.9 \\
Middle Eastern & 32.4 & 23.2 & 6.2 & 8.2 & 4.6 \\
South/Central Asian & 31.8 & 21.6 & 3.8 & 5.0 & 2.8 \\
East Asian & 16.2 & 11.9 & 3.7 & 4.9 & 2.7 \\
Asian & 24.0 & 16.7 & 3.8 & 5.0 & 2.8 \\
Oceanian & 48.0 & 33.4 & 7.3 & 9.6 & 5.4 \\
\hline
\end{tabular}

in Steps 3-5. Applying these ethnicity-based estimates to each state/province/territories' unique ethnic distributions, we showed the same pattern across all US states (Table 2) and Canada provinces/territories (Table 3). We estimated that about one-third of individuals entering the $\mathrm{STAR}^{*} \mathrm{D}$ algorithm in the US (mean $=32.2 \%, \mathrm{SD}=1.4 \%$, range $=23.5-33.2 \%$ ) and Canada (mean $=29.4 \%$, $\mathrm{SD}=4.5 \%$, range $=19.3-34.5 \%)$ could benefit from $C Y$ P2C19 genotyping. Whereas, approximately one-fifth of individuals progressing to Step 2 in the US (mean $=22.6 \%$, $\mathrm{SD}=1.0 \%$, range $16.4-23.3 \%$ ) and Canada (mean $=20.6 \%$, $\mathrm{SD}=3.2 \%$, range $13.6-24.2 \%)$ would benefit from $C Y$ P2C19 or CYP2D6 genotyping. Separate estimates for CYP2C19 and CYP2D6 in Step 2 showed on average 19.9\% $(\mathrm{SD}=0.9$, range $=14.5-20.5 \%)$ and $18.1 \%(\mathrm{SD}=2.8 \%$, range $=11.9-21.3 \%)$ of those in the US and Canada, respectively, would benefit from CYP2C19 genotyping, while on average an additional $2.8 \%$ ( $\mathrm{SD}=0.2 \%$, range $1.9-3.0 \%$ ) and $2.5 \%(\mathrm{SD}=0.4 \%$, range $=1.7-2.9 \%)$ in the US and Canada, respectively, would benefit from CYP2D6 genotyping. For individuals progressing to Steps 3 , 4, or 5 of the STAR ${ }^{*}$ algorithm, we estimated that less than $10 \%$ at each of these steps would benefit from CYP2D6 genotyping.

\section{Discussion/Conclusion}

Our findings suggest a potential benefit of combining protocol-based depression care with CYP2C19 and CY$P 2 D 6$ genotyping in the US and Canada. The potential impact of CYP2C19 genotyping was clearly seen within the first two steps of the STAR*D treatment algorithm, whereas, the potential impact of CYP2D6 genotyping was most notable in Steps 3, 4, and 5.
We estimated that up to one-third of individuals entering Steps 1 and 2 of the STAR*D treatment algorithm could benefit from CYP2C19 genotyping. This estimated impact is a direct reflection of the number of treatment options in Steps 1 and 2 that are linked to CYP2C19-based prescribing guidelines. In Step 1, citalopram is the only option and in Step 2 citalopram combination therapies and sertraline comprise four of the six pharmacotherapy options. Both citalopram and sertraline have CYP2C19based prescribing guidelines that recommend dose reductions or alternative medications for individuals that are CYP2C19 poor or rapid/ultrarapid metabolizers, respectively [6]. As such, the first two steps of the STAR*D algorithm are not ideally matched for CYP2C19 poor, rapid, or ultrarapid metabolizers, a group of individuals that represents $21-48 \%$ of any given population depending on ethnicity distributions [9]. Not accounting for this considerable proportion of the population when using the STAR*D algorithm could unnecessarily expose these individuals to antidepressant trials for which the likelihood of an undesirable outcome is increased. However, if these individuals were identified prior to entering the algorithm, it would be reasonable for a clinician to prescribe venlafaxine (assuming normal CYP2D6 metabolism) or bupropion first as they are the only two pharmacotherapy options in the first two steps that are not implicated in a CYP2C19-based prescribing guideline.

Beyond the first two steps of the STAR*D algorithm, the benefits of CYP2C19 genotyping gives way to $C Y$ P2D6 genotyping. In Steps 3-5, nortriptyline and/or venlafaxine are included as treatment options, both of which have CYP2D6-based prescribing guidelines $[4,5]$. However, unlike Steps 1 and 2, in the final three steps of the STAR*D algorithm, the proportion of treatment options with pharmacogenetic-based prescribing guidelines is lower. As a result, the potential benefits of CYP2D6 genotyping in conjunction with the STAR*D algorithm was estimated to be modest. Nevertheless, the estimated proportion (2.5-7.7\%) of individuals entering these steps that could benefit from CYP2D6 genotyping is not trivial.

Collectively, these results suggest that CYP2C19 and CYP2D6 genetic testing would be beneficial for a considerable proportion of population entering the STAR*D treatment algorithm, regardless of which state, province, or territory the care is being provided. That said, our estimates, as expected, did vary slightly by geography. This geographical variation can be attributed to differences in the proportion of ethnic groups represented in each state, province, and territory we examined. For example, CPIC estimates that $34 \%$ of individuals who identify as Cauca-
Fan/Bousman 
Table 2. Estimated percentage of individuals with a CYP2C19 and/ or CYP2D6 phenotype contraindicated for the treatment received at each step of the STAR*D algorithm in the United States

\begin{tabular}{|c|c|c|c|c|c|}
\hline State & Step 1 & Step 2 & Step 3 & Step 4 & Step 5 \\
\hline Alabama & 32.6 & 23.1 & 5.7 & 7.5 & 4.2 \\
\hline Alaska & 29.9 & 20.9 & 4.9 & 6.4 & 3.6 \\
\hline Arizona & 32.2 & 22.6 & 5.3 & 7.0 & 3.9 \\
\hline Arkansas & 32.8 & 23.1 & 5.5 & 7.3 & 4.1 \\
\hline California & 31.0 & 21.7 & 5.1 & 6.7 & 3.8 \\
\hline Colorado & 32.5 & 22.8 & 5.3 & 7.0 & 3.9 \\
\hline Connecticut & 32.5 & 22.8 & 5.4 & 7.1 & 4.0 \\
\hline Delaware & 32.2 & 22.7 & 5.5 & 7.3 & 4.1 \\
\hline District of Columbia & 31.5 & 22.4 & 5.8 & 7.7 & 4.3 \\
\hline Florida & 32.6 & 22.9 & 5.5 & 7.3 & 4.1 \\
\hline Georgia & 32.1 & 22.7 & 5.7 & 7.5 & 4.2 \\
\hline Hawaii & 23.5 & 16.4 & 3.7 & 4.9 & 2.8 \\
\hline Idaho & 32.9 & 23.0 & 5.3 & 7.0 & 3.9 \\
\hline Illinois & 32.4 & 22.8 & 5.4 & 7.2 & 4.0 \\
\hline Indiana & 32.9 & 23.1 & 5.4 & 7.2 & 4.0 \\
\hline Iowa & 33.0 & 23.1 & 5.4 & 7.1 & 4.0 \\
\hline Kansas & 32.5 & 22.8 & 5.3 & 7.0 & 3.9 \\
\hline Kentucky & 33.0 & 23.2 & 5.4 & 7.2 & 4.0 \\
\hline Louisiana & 32.5 & 23.0 & 5.7 & 7.6 & 4.2 \\
\hline Maine & 33.2 & 23.3 & 5.4 & 7.1 & 4.0 \\
\hline Maryland & 31.7 & 22.4 & 5.6 & 7.4 & 4.1 \\
\hline Massachusetts & 32.3 & 22.7 & 5.3 & 7.0 & 3.9 \\
\hline Michigan & 32.5 & 22.9 & 5.5 & 7.2 & 4.0 \\
\hline Minnesota & 32.4 & 22.8 & 5.3 & 7.0 & 3.9 \\
\hline Mississippi & 32.6 & 23.1 & 5.8 & 7.7 & 4.3 \\
\hline Missouri & 32.8 & 23.0 & 5.5 & 7.2 & 4.0 \\
\hline Montana & 32.5 & 22.8 & 5.3 & 6.9 & 3.9 \\
\hline Nebraska & 32.8 & 23.0 & 5.4 & 7.1 & 4.0 \\
\hline Nevada & 31.5 & 22.1 & 5.2 & 6.9 & 3.8 \\
\hline New Hampshire & 33.1 & 23.2 & 5.4 & 7.1 & 4.0 \\
\hline New Jersey & 31.9 & 22.4 & 5.4 & 7.1 & 4.0 \\
\hline New Mexico & 32.2 & 22.5 & 5.2 & 6.9 & 3.9 \\
\hline New York & 31.8 & 22.4 & 5.4 & 7.1 & 4.0 \\
\hline North Carolina & 32.4 & 22.8 & 5.6 & 7.3 & 4.1 \\
\hline North Dakota & 32.7 & 22.9 & 5.3 & 7.0 & 3.9 \\
\hline Ohio & 32.7 & 23.0 & 5.5 & 7.2 & 4.0 \\
\hline Oklahoma & 30.9 & 21.7 & 5.1 & 6.7 & 3.8 \\
\hline Oregon & 32.2 & 22.5 & 5.2 & 6.9 & 3.9 \\
\hline Pennsylvania & 32.7 & 23.0 & 5.4 & 7.2 & 4.0 \\
\hline Rhode Island & 32.5 & 22.8 & 5.4 & 7.1 & 4.0 \\
\hline South Carolina & 32.6 & 23.0 & 5.7 & 7.5 & 4.2 \\
\hline South Dakota & 32.4 & 22.7 & 5.3 & 6.9 & 3.9 \\
\hline Tennessee & 32.8 & 23.1 & 5.5 & 7.3 & 4.1 \\
\hline Texas & 32.5 & 22.9 & 5.4 & 7.2 & 4.0 \\
\hline Utah & 32.9 & 23.0 & 5.3 & 7.0 & 3.9 \\
\hline Vermont & 33.1 & 23.2 & 5.4 & 7.1 & 4.0 \\
\hline Virginia & 31.9 & 22.5 & 5.4 & 7.2 & 4.0 \\
\hline Washington & 31.4 & 22.0 & 5.1 & 6.8 & 3.8 \\
\hline West Virginia & 33.2 & 23.3 & 5.4 & 7.2 & 4.0 \\
\hline Wisconsin & 32.9 & 23.0 & 5.4 & 7.1 & 4.0 \\
\hline Wyoming & 33.0 & 23.1 & 5.3 & 7.1 & 3.9 \\
\hline Average & 33.2 & 22.6 & 5.4 & 7.1 & 4.0 \\
\hline Standard deviation & 1.4 & 1.0 & 0.3 & 0.4 & 0.2 \\
\hline
\end{tabular}

Impact of Pharmacogenetic Testing on Protocol-Based Care
Table 3. Estimated percentage of individuals with a CYP2C19 and/ or CYP2D6 phenotype contraindicated for the treatment received at each step of the STAR*D algorithm in Canada

\begin{tabular}{lrrrrr}
\hline Province/territory & \multicolumn{5}{c}{ Step 1 Step 2 Step 3 Step 4 Step 5 } \\
\hline Alberta & 29.9 & 21.0 & 5.0 & 6.6 & 3.7 \\
British Columbia & 30.5 & 21.4 & 5.1 & 6.7 & 3.7 \\
Manitoba & 33.3 & 23.4 & 5.5 & 7.3 & 4.1 \\
New Brunswick & 24.2 & 17.0 & 4.0 & 5.2 & 2.9 \\
Newfoundland and Labrador & 23.7 & 16.6 & 3.9 & 5.1 & 2.9 \\
Northwest Territories & 32.3 & 22.7 & 5.4 & 7.1 & 4.0 \\
Nova Scotia & 28.9 & 20.3 & 4.8 & 6.3 & 3.5 \\
Nunavut & 33.2 & 23.3 & 5.5 & 7.3 & 4.1 \\
Ontario & 30.9 & 21.7 & 5.1 & 6.8 & 3.8 \\
Prince Edward Island & 28.0 & 19.6 & 4.6 & 6.1 & 3.4 \\
Quebec & 19.3 & 13.6 & 3.3 & 4.4 & 2.5 \\
Saskatchewan & 32.9 & 23.1 & 5.4 & 7.1 & 4.0 \\
Yukon & 34.5 & 24.2 & 5.7 & 7.5 & 4.2 \\
Average & $\mathbf{2 9 . 4}$ & $\mathbf{2 0 . 6}$ & $\mathbf{4 . 9}$ & $\mathbf{6 . 4}$ & $\mathbf{3 . 6}$ \\
Standard deviation & $\mathbf{4 . 5}$ & $\mathbf{3 . 2}$ & $\mathbf{0 . 7}$ & $\mathbf{1 . 0}$ & $\mathbf{0 . 5}$ \\
\hline
\end{tabular}

sian have a CYP2C19 actionable phenotype (i.e., poor, rapid, or ultrarapid metabolizer), second only to those of Oceanian descent (48\% actionable) [9]. However, Oceanians represented a very minor proportion of the populations in Canada and in the US and as such jurisdictions with a greater proportion of Caucasians (e.g., Yukon, West Virginia, and Maine) were estimated to have the greatest proportion of individuals who could benefit from CYP2C19 testing at Steps 1 and 2. This also explains why Hawaii, which had a relatively small Caucasian population, had the smallest proportion of individuals estimated to benefit from CYP2C19 testing at Steps 1 and 2, despite having a relatively larger proportion of individuals of Oceanian descent.

There are several notable limitations to consider when interpreting our findings. First, we assumed that the ethnicities of antidepressant recipients were identical to the ethnic group proportions in each state, territory, and province. Second, we held rates of antidepressant use within each country constant across geographic locations, ethnic groups, age, and sex, as the data required to adequately adjust our simulations to these factors were not available. These assumptions could result in under- or over-estimations of the potential benefit of CYP2C19 and CYP2D6 genotyping, depending on the distribution of these demographic factors in each state, province, or territory. Third, the ethnic categories were based on Canadian or US census data. Due to differences in the collection and classification of census 
ethnicity data, the results for Canada and the US cannot be directly compared. Fourth, the ethnic categories used by the Canadian or US census data were not identical to the categories used by CPIC to report CYP2C19 and CY$P 2 D 6$ phenotype frequencies. This meant that we were unable to capture and estimate the CYP2C19 and $C Y$ P2D6 phenotypes for some self-reported ethnic origins (e.g., Acadian, two or more races). Notably, about half of the Quebec population identified solely as "Canadian." Thus, our estimates for this province are limited and should be interpreted with caution. Fourth, it is not clear whether our estimates are generalizable to other protocol-based care algorithms or guidelines for depression such as the Texas Medication Algorithm Project (TMAP) [14] or the Canadian Network for Mood and Anxiety Treatments (CANMAT) Guidelines [15]. However, $60 \%$ and $40 \%$ of first-line medications recommended by TMAP and CANMAT, respectively, are linked to a CYP2C19- or CYP2D6-based prescribing guideline, suggesting that similar results might be observed if these protocols were to be systematically examined. Fifth, the cost-effectiveness of pairing CYP2C19 and CYP2D6 with protocol-based care has not been evaluated. However, economic analyses of combinatorial pharmacogenetic testing (including CYP2C19 and CYP2D6) to guide antidepressant prescribing have suggested that this testing - on its own - is a cost-effective strategy $[16,17]$. Sixth, the phenotype frequencies we used assume that high-quality genotyping of common functional CYP2C19 and CYP2D6 variants is performed. That said, genotyping for rare functional variants in $C Y$ P2C19 and CYP2D6 is not currently standard practice. Previous work has shown that rare variants comprise 4.4 and $6.3 \%$ of all functional variants in CYP2C19 and CY$P 2 D 6$, respectively [18]. As such, our results are likely an underestimate of the true proportion of individuals that could benefit from pharmacogenetic testing. Finally, our results and interpretations are constrained to the medications that are included in the STAR*D algorithm and do not account for concomitant or future medications that an individual may receive. As such, the estimated benefits of CYP2C19 and CYP2D6 genotyping are likely underestimated given that guidelines associated with these genes are also available for other antidepressants (i.e., escitalopram, fluvoxamine, paroxetine, amitriptyline, clomipramine, imipramine, doxepin, desipramine, trimipramine), antipsychotics (i.e., aripiprazole, haloperidol, pimozide, zuclopenthixol), and the ADHD medication atomoxetine [19]. On the other hand, it could be argued that commonly used prescrib- ing strategies such as "start low and go slow" would in part mitigate tolerability concerns among those with CYP2C19 and/or CYP2D6 reduced function phenotypes (e.g., poor metabolizers), suggesting that our findings overestimate the potential benefit of CYP2C19 and CY$P 2 D 6$ testing. However, generalized strategies like "start low and go slow" can be problematic for individuals that have increased function phenotypes (i.e., rapid and ultrarapid), increasing the time to reach therapeutic plasma concentrations and symptom remission. Nevertheless, the current study cannot reconcile these arguments and as such future prospective or retrospective trials are needed to refine the estimates provided in the current study.

Despite these limitations, our findings support the simultaneous implementation of CYP2C19 and CYP2D6 genetic testing alongside the $\mathrm{STAR}^{*} \mathrm{D}$ treatment algorithm. We estimated that up to one-third of the US and Canadian populations being treated for depression could benefit from the addition of CYP2C19 and CYP2D6 genetic testing. This is aligned with what we already know from the STAR*D trial results that showed implementation of the algorithm alone fails to get one-third of individuals well [2] and that common pharmacogenetic variation may play a role in patient outcomes [3]. Although future experimental research is required to validate our estimates and determine the cost-effectiveness of adding CYP2C19 and CYP2D6 genetic testing to protocol-based depression care, it seems reasonable to conclude that the addition of such testing has tremendous potential to assist clinicians in the selection of appropriate therapies and reduction of the number of medication trials a patient must endure before getting well.

\section{Statement of Ethics}

This study used publicly available, deidentified, aggregate data and as such was deemed exempt from ethics review.

\section{Disclosure Statement}

M.F. has no conflicts of interest to declare. C.A.B. has received material support from Assurex Health (Myriad Neuroscience), CNSDose, Genomind, and AB-Biotics for research purposes and has ongoing research collaborations with MyDNA but does not have equity, stocks, or options in these companies or any other pharmacogenetic companies. C.A.B. is a member of the Clinical Pharmacogenetics Implementation Consortium and the Genetic Testing Committee of the International Society of Psychiatric Genetics.
Fan/Bousman 


\section{Funding Sources}

The authors did not receive any funding.

\section{Author Contributions}

C.A.B. designed and planned the analysis for the study. M.F. designed and conducted the simulations. Both authors wrote the manuscript.

\section{References}

1 Rush AJ, Fava M, Wisniewski SR, Lavori PW, Trivedi MH, Sackeim HA, et al.; STAR*D Investigators Group. Sequenced treatment alternatives to relieve depression $\left(\mathrm{STAR}^{*} \mathrm{D}\right)$ : rationale and design. Control Clin Trials. 2004 Feb;25(1):119-42.

2 Rush AJ, Trivedi MH, Wisniewski SR, Nierenberg AA, Stewart JW, Warden D, et al. Acute and longer-term outcomes in depressed outpatients requiring one or several treatment steps: a STAR*D report. Am J Psychiatry. 2006 Nov; 163(11):1905-17.

3 Mrazek DA, Biernacka JM, O’Kane DJ, Black JL, Cunningham JM, Drews MS, et al. CYP2C19 variation and citalopram response. Pharmacogenet Genomics. 2011 Jan;21(1): 1-9.

4 Swen JJ, Nijenhuis M, de Boer A, Grandia L, Maitland-van der Zee AH, Mulder $\mathrm{H}$, et al. Pharmacogenetics: from bench to byte-an update of guidelines. Clin Pharmacol Ther. 2011 May;89(5):662-73.

5 Hicks JK, Sangkuhl K, Swen JJ, Ellingrod VL, Müller DJ, Shimoda K, et al. Clinical pharmacogenetics implementation consortium guideline (CPIC) for CYP2D6 and CYP2C19 genotypes and dosing of tricyclic antidepressants: 2016 update. Clin Pharmacol Ther. 2017 Jul;102(1):37-44.

6 Hicks JK, Bishop JR, Sangkuhl K, Müller DJ, Ji Y, Leckband SG, et al.; Clinical Pharmacogenetics Implementation Consortium. Clinical Pharmacogenetics Implementation Consortium (CPIC) guideline for CYP2D6 and CYP2C19 genotypes and dosing of selective serotonin reuptake inhibitors. Clin Pharmacol Ther. 2015 Aug;98(2):127-34.
7 Statistics Canada. Census Profile, 2016 Census [Internet]. Government of Canada, Statistics Canada. 2019 [cited 2019 Jun 13]. Available from: https://www12.statcan.gc.ca/census-recensement/2016/dp-pd/prof/index. cfm?Lang=E

8 American Community Survey Office. 2016 Data Profiles [Internet]. 2016 Data Profiles | American Community Survey | U.S. Census Bureau. 2017 [cited 2019 Jun 13]. Available from: https://www.census.gov/acs/www/ data/data-tables-and-tools/data-profiles/2016/

9 Relling MV, Klein TE. CPIC: clinical Pharmacogenetics Implementation Consortium of the pharmacogenomics research network. Clin Pharmacol Ther. 2011 Mar; 89(3):464-7.

10 Gaedigk A, Sangkuhl K, Whirl-Carrillo M, Twist GP, Klein TE, Miller NA; PharmVar Steering Committee. The Evolution of PharmVar. Clin Pharmacol Ther. 2019 Jan;105(1): 29-32.

11 Caudle KE, Dunnenberger HM, Freimuth RR, Peterson JF, Burlison JD, Whirl-Carrillo $\mathrm{M}$, et al. Standardizing terms for clinical pharmacogenetic test results: consensus terms from the Clinical Pharmacogenetics Implementation Consortium (CPIC). Genet Med. 2017 Feb;19(2):215-23.

12 OECD. Antidepressant drugs consumption, 2000 and 2015 (or nearest year). Health at a Glance. 2017;2017:1-216.

13 Pratt LA, Brody DJ, Gu Q. Antidepressant use among persons aged 12 and over: United States, 2011-2014. NCHS Data Brief. 2017 Aug;(283):1-8
14 Trivedi MH, Rush AJ, Crismon ML, Kashner TM, Toprac MG, Carmody TJ, et al. Clinical results for patients with major depressive disorder in the Texas Medication Algorithm Project. Arch Gen Psychiatry. 2004 Jul;61(7): 669-80.

15 Kennedy SH, Lam RW, McIntyre RS, Tourjman SV, Bhat V, Blier P, et al.; CANMAT Depression Work Group. Canadian Network for Mood and Anxiety Treatments (CANMAT) 2016 Clinical Guidelines for the Management of Adults with Major Depressive Disorder: Section 3. Pharmacological Treatments. Can J Psychiatry. 2016 Sep;61(9):540-60.

16 Fagerness J, Fonseca E, Hess GP, Scott R, Gardner KR, Koffler M, et al. Pharmacogenetic-guided psychiatric intervention associated with increased adherence and cost savings. Am J Manag Care. 2014 May;20(5):e146-56.

17 Brown LC, Lorenz RA, Li J, Dechairo BM. Economic Utility: Combinatorial Pharmacogenomics and Medication Cost Savings for Mental Health Care in a Primary Care Setting. Clin Ther. 2017 Mar;39(3):592-602.e1.

18 Ingelman-Sundberg M, Mkrtchian S, Zhou Y, Lauschke VM. Integrating rare genetic variants into pharmacogenetic drug response predictions. Hum Genomics. 2018 May; 12(1):26.

19 Bousman CA, Zierhut H, Müller DJ. Navigating the labyrinth of pharmacogenetic testing: A guide to test selection. Clin Pharmacol Ther. 2019 Aug;106(2):309-12. 